\title{
Cardiac computed tomography radiomics: an emerging tool for the non-invasive assessment of coronary atherosclerosis
}

\author{
Alessandro Murgia ${ }^{1}$, Antonella Balestrieri ${ }^{1}$, Paola Crivelli ${ }^{2}$, Jasjit S. Suri ${ }^{3}$, Maurizio Conti ${ }^{2}$, \\ Filippo Cademartiri ${ }^{4}$, Luca Saba ${ }^{1}$ \\ ${ }^{1}$ Department of Radiology, University of Cagliari, Cagliari CA, Italy; ${ }^{2}$ Department of Radiology, University of Sassari, Sassari SS, Italy; ${ }^{3}$ Stroke and \\ Monitoring Division, AtheroPoint ${ }^{\mathrm{TM}}$, Roseville, CA, USA; ${ }^{4} \mathrm{SDN}$, Naples, Italy \\ Contributions: (I) Conception and design: F Cademartiri, L Saba; (II) Administrative support: All authors; (III) Provision of study materials or patients: \\ All authors; (IV) Collection and assembly of data; All authors; (V) Data analysis and interpretation: JS Suri, M Conti, F Cademartiri, L Saba; (VI) \\ Manuscript writing: All authors; (VII) Final approval of manuscript: All authors. \\ Correspondence to: Luca Saba. Department of Radiology, University of Cagliari, Cagliari, Italy. Email: lucasabamd@gmail.com.
}

\begin{abstract}
In the last decades, significant advances have been made in the preventive approaches to cardiovascular disease. Even so, coronary artery disease remains one of the main causes of morbidity and mortality worldwide. Invasive imaging modalities, such as intravascular ultrasound or optical coherence tomography, have played a key role in the comprehension of the pathological processes underlying myocardial infarction and cerebrovascular disease. These imaging techniques have contributed greatly to the identification and phenotyping of the culprit lesion, the so-called vulnerable plaque. Coronary computed tomographic angiography (CCTA) has emerged in more recent years as the non-invasive modality of choice in the study of coronary atherosclerosis, showing in many studies a diagnostic yield comparable to invasive approaches. Moreover, being able to describe extra-luminal characteristics of the affected vessel, CCTA has greatly contributed towards shifting the attention of researchers from the mere quantification of luminal stenosis to the identification of adverse plaque features, which appear to have a stronger prognostic value. However, the identification of some of the hallmarks of vulnerable plaques is qualitative in nature and, therefore, subject to some degree of inter-reader variability. Moreover, CCTA is still unable to identify some fine markers of plaque vulnerability which can be detected by invasive techniques, such as neovascularization and plaque erosion, among others. Nonetheless, radiological images can be viewed as vast 3-D datasets which, via the use of recent technology, allow for the extraction of numerous quantitative features that may be used to accurately phenotype a given lesion. Radiomics is the process of extrapolating innumerable parameters from a given region of interest, with the goal of establishing correlations between quantitative variables and clinical data. These datasets can then be manipulated to create predictive models via the use of automated algorithms in a process called machine learning. As a result of these approaches, radiological images may offer information regarding the characterization of a plaque which can go much beyond the boundaries of what can be qualitatively asserted by the human eye, contributing to expanding the knowledge of the disease and ultimately assist clinical decisions. Thus far, radiomics has found its more consistent area of application in the field of oncology; to present date, the amount of clinical data regarding coronary artery disease is still relatively small, partly due to the technical difficulties associated with the implementation of such techniques to the study of a small and geometrically complex lesion such as the coronary plaque. The present review, after a summary of the imaging modalities most commonly used nowadays in the study of coronary plaques, will provide a perspective on the application of radiomic analysis to coronary artery disease.
\end{abstract}

Keywords: Imaging modalities; coronary plaques; coronary atherosclerosis; radiomics

Submitted Feb 03, 2020. Accepted for publication Jul 08, 2020.

doi: $10.21037 /$ cdt-20-156

View this article at: http://dx.doi.org/10.21037/cdt-20-156 


\section{Atherosclerosis and cardiovascular disease}

Atherosclerosis is an inflammatory vascular disease caused by lipidic and other metabolic alterations. The most relevant manifestation of atherosclerosis is cardiovascular disease, which results in two major conditions: ischemic heart disease and cerebrovascular disease. Even though the second half of the $20^{\text {th }}$ century has witnessed a significant reduction in the incidence and mortality rates connected to cardiovascular disease in high-income countries (Figure 1), atherosclerosis still represents the main cause of mortality worldwide, with ischemic heart disease and stroke accounting for 247.9 deaths in 100,000 persons in $2013(1,2)$.

The pathological process of atherosclerosis is characterized by lipid deposition and inflammatory changes to vessel wall, with coronary arteries being involved in this process to some degree almost invariably from middle age onwards. These lesions can cause progressive stenosis of the coronary lumen or, alternatively, determine plaque rupture or erosion with subsequent thrombosis and acute myocardial infarction. Imaging techniques play a role of vital importance in understanding and monitoring the processes which underlie acute adverse events such as plaque rupture and erosion.

Schematically, a number of subsequent steps can be identified in the progression of coronary atherosclerosis: first the deposition of LDL within the arterial intima, lipid oxidation, inflammatory response led by lipid-filled macrophages (foam cell formation), and progression of inflammatory response towards proteolysis and cell death and, ultimately, the formation of unstable coronary lesions predisposed to acute plaque rupture. Unstable plaques frequently display characteristic histopathology: a large necrotic core, a thin fibrous cap, positive remodeling and microcalcifications (the so-called thin-cap fibroatheroma).

The natural history of an atherosclerotic plaque depends on a sensitive balance between pro- and anti-inflammatory effects. It has been demonstrated that smooth muscle cell migration from the arterial media plays a central role in the stabilization of a plaque via the secretion of fibrous matrix components that thicken and strengthen the fibrous cap. Calcification also represents a healing response to the inflammatory insult, although it acts in a bi-modal fashion: microcalcification in the initial stages correlates to unstable plaques, whereas macroscopic calcification in the later stages provides stability (3).

Despite the knowledge acquired in the pathophysiology of coronary atherosclerosis, the identification of lesions at

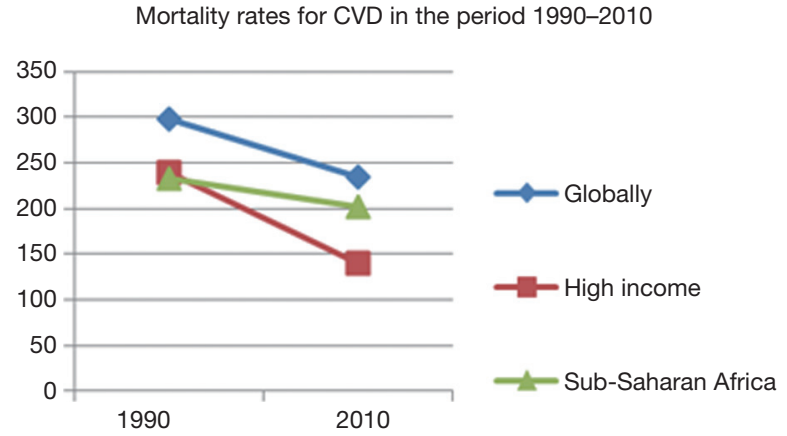

Figure 1 Age-standardized cardiovascular mortality rates per 100,000 inhabitants in 1990 and 2010 worldwide, in high income countries and in Sub-Saharan countries. Line chart demonstrates a global reduction in mortality rates for CVD, with a more steep trend for high-income countries compared to lower income countries (in this case Sub-Saharan Africa) in the same period (approximately 40\% vs. 13\%). Readapted from: Barquera et al. (1).

risk of erosion still represents a challenging task. Indeed, current imaging technology has only partially proven successful in identifying unstable plaques, which poses a severe limit to the capability of clinicians to stratify risk. Therefore, further research is urgently needed in this field in order to identify novel diagnostic approaches.

\section{Imaging of the atherosclerotic coronary arteries}

The imaging of coronary artery disease (CAD) comprises a vast armamentarium of diagnostic tools which, broadly speaking, can provide detail on: anatomic and physiological aspects, plaque composition, molecular activity and biomechanical stresses (4). The present section will provide a general overview of the main imaging tools available nowadays to characterize coronary atherosclerotic plaques; it will then focus on the role played by coronary computed tomography angiography (CCTA), its limitations and future directions.

\section{CAD: a wide array of diagnostic imaging tools}

The gold-standard for the diagnosis of CAD is still nowadays represented by invasive angiography. This procedure involves the catheterization of the coronary ostia via a peripheral arterial access, with subsequent injection of radio-opaque contrast and visualization of the vessel anatomy under X-ray fluoroscopy. Angiography offers unmatched spatial and temporal resolution $(0.1-0.2 \mathrm{~mm}$ 
and $10 \mathrm{~ms}$ respectively) and is therefore an unsurpassed methodology in the description of the degree of coronary luminal stenosis; furthermore, it is the imaging technique of choice when revascularization procedures are contemplated.

However, recent research indicates that luminal stenosis alone cannot be considered a reliable marker for hemodynamic obstruction or accurately evaluate the ischemic burden. In case of persistent angina despite optimal medical therapy, functional tests provide more information than mere anatomic assessment; indeed, the hemodynamic significance of a plaque has proven a reliable predictor of acute cardiovascular events $(5,6)$. Hemodynamically significant CAD can be evaluated noninvasively (stress echocardiography, stress perfusion MR, myocardial perfusion SPECT or PET, CT perfusion) or invasively (fractional flow reserve during angiography). Fractional flow reserve (FFR) is the most widely accepted functional method, as it provides a reliable pressure-based assessment of relative coronary flow reserve across the atherosclerotic plaque (7).

Noninvasive angiography can be achieved via computed tomography (coronary computed tomography angiography) or magnetic resonance (magnetic resonance angiography, MRA), provided ECG-gating is conducted to eliminate artefacts of cardiac movement (4).

CCTA is preferred over MRA for anatomic coronary evaluation. New generation CT scanners can offer spatial resolution as high 0.5 to $0.6 \mathrm{~mm}$ with a temporal resolution of 66 to $210 \mathrm{~ms}$. In the presence of angina with a lowto-moderate risk of CAD, CCTA performs well as a first-line diagnostic tool thanks to an excellent negative predictive value, although quantification of stenosis can be overestimated owing to artefacts caused by coronary calcification. In this respect, dual-energy CT renders a better coronary plaque characterization by increasing tissue contrast, even though at the expense of a poorer image quality (8).

CCTA is often performed in tandem with coronary artery calcification (CAC) scoring, a technique that provides an estimate of the overall disease burden. CAC has historically been used to stratify the risk for CAD, although there is no consistent evidence in the literature that it may effectively be used to monitor the therapeutic response over time. Newer approaches that discriminate calcium density from volume may offer more favorable results, following the rationale that higher density coronary calcification may contribute to plaque stabilization (9-11).

Compared with CT, MRI allows for better soft-tissue characterization and avoids radiation exposure, being therefore suitable for children or pregnant women. Its main application remains the study of large, static vessels (carotids, aorta, peripheral vessels), while the inspection of coronary circulation remains challenging due to an unsatisfactory control of motion artefacts, contrast-to-noise ratio, spatial resolution and volumetric coverage. However, coronary MRI technology is rapidly evolving and can reliably image proximal and midvessels; in the future, its limitations may be overcome and allow for a comprehensive evaluation of coronary anatomy, ventricular wall motion, myocardial perfusion, viability and scarring.

There is growing evidence in the literature that, beyond the degree of luminal stenosis, a number of factors connected with plaque composition and morphology play a pivotal role in causing cardiovascular acute events. Recent research has focused on the identification of clinical biomarkers and imaging features of "vulnerable" or "unstable" plaques, similarly to what was found for the carotid artery bed (12-14). In this regard, the thincap fibroatheroma is generally recognized as the most reliable precursor lesion of plaque acute events. As far as invasive approaches are considered, the imaging techniques available to demonstrate plaque morphology are: intravascular coronary imaging with ultrasound (IVUS), optical coherence tomography (OCT) and near infraredspectroscopy (NIRS). For what concerns non-invasive counterparts, CCTA represents an exceptional tool thanks to its capability to deliver information not only on coronary anatomy and stenosis, but also on plaque morphology and composition.

With regards to nuclear medicine techniques, positron emission tomography (PET) is a non-invasive modality that exploits molecularly targeted probes conjugated to a radioactive isotope which can illuminate various plaque features. Long scan times, low spatial resolution and not well-spread availability have traditionally posed a challenge to the diffusion of coronary PET imaging; the introduction of hybrid PET-CT and PET-MR systems have partly addressed these issues. Most clinical research has thus far focused on three types PET tracers: ${ }^{18} \mathrm{~F}$-Fluorodeoxyglucose $\left({ }^{18} \mathrm{~F}\right.$-FDG $),{ }^{68}$ Gallium-labelled DOTATATE $\left({ }^{68}\right.$ GalliumDOTATATE) and sodium fluoride labeled with fluorine ${ }^{18}$ $\left({ }^{18} \mathrm{~F}-\mathrm{NaF}\right)(15)$.

${ }^{18} \mathrm{~F}-\mathrm{FDG}$ is an unspecific marker of inflammation which acts by detecting areas of increased macrophage activity. In the carotid artery bed, ${ }^{18} \mathrm{~F}-\mathrm{FDG}$ uptake has been shown to correlate with the presence of high-risk plaque 
features and to identify post-treatment reduction of plaque inflammation (16). While similar results may reasonably be expected for the coronary artery bed, in practice intense myocardial uptake obscures coronary signal, making the use of this tracer unpractical for CAD diagnostics.

${ }^{68}$ Gallium-DOTATATE is a marker of inflammation which acts by binding to somatostatin receptor subtype 2 (SSTR2) on macrophages. In comparison to ${ }^{18} \mathrm{~F}-\mathrm{FDG}$, myocardial uptake appears to be minimal, thus enabling for a more reliable identification of culprit coronary (and carotid) plaques (17).

Recent research indicates that ${ }^{18} \mathrm{~F}-\mathrm{NaF}$ can highlight active mineralization by binding to exposed surface of hydroxyapatite, thus documenting microcalcific deposits, a recognized feature of plaque instability, prior to the identification of macrocalcification at CCTA. An ongoing prospective multicentre trial will bring further evidence regarding the ability of ${ }^{18} \mathrm{~F}-\mathrm{NaF}$ to improve risk stratification in post-infarct patients (NCT02278211).

Finally, another target for the imaging evaluation of coronary atherosclerosis is represented by the analysis of vascular wall shear stress, a well-known predisposing condition to the formation of atherosclerotic plaques. Wall shear stress can be estimated via computational simulations that calculate coronary blood-flow through a $3 \mathrm{D}$ reconstruction of the vascular tree. A number of factors are taken into account such as blood pressure, plaque morphology, plaque composition and luminal geometry. This can be achieved both via CCTA or intravascular imaging combined with biplane angiography. The same concept lies behind CT-calculated fractional flow reserve (CT-FFR), which in several clinical studies showed comparable results to the invasive FFR in CAD with a degree of stenosis higher that $50 \%(18,19)$.

\section{The role of CCTA in CAD}

As it was previously discussed, CCTA is an imaging tool of widely recognized importance in the evaluation of $\mathrm{CAD}$, as it represents a non-invasive and cost-effective alternative to other imaging modalities.

In its early days CCTA was merely considered the noninvasive counterpart of invasive coronary angiography, leading most research to concentrate on the clinical predictive value of luminal stenosis found on CTA. In this respect, according to the Society of Cardiovascular Computed Tomography (SCCT), luminal stenosis can be described as minimal (<25\%), mild ( $25 \%$ to $49 \%)$, moderate
( $50 \%$ to $69 \%$ ), severe ( $70 \%$ to $99 \%$ ) and occluded; a cut-off of $50 \%$ to $70 \%$ has been found to correlate significantly to adverse later outcomes (20).

However, CCTA derives its strength from the fact that it is not only capable of evaluating the degree of stenosis, but it also allows for the description of the wall characteristics and the morphology of the atherosclerotic plaque.

The ability to evaluate plaque morphology and composition resulted in the identification of high-risk plaque features, which retain a higher descriptive value than mere stenosis. Thus, unstable lesions tend be large in volume, non-calcified, low attenuating, with spotty calcifications and of a higher remodeling index (21). It is significantly important that such markers predict major adverse cardiac events (MACE) even in the presence of nonobstructive lesions. Plaque volume is closely connected to positive (or outward) remodeling, which can be seen as an adaptive response to plaque progression, acting to preserve luminal caliber at the expense of vessel wall enlargement. Importantly, positive remodeling is typically associated to the presence of a large lipid core and high macrophage density (threshold for positive remodeling: crosssectional area $10 \%$ higher than reference segment; spotty calcification: diameter less than $3 \mathrm{~mm}$ in all directions). Another sign which strongly associates with MACE is the napkin-ring sign (NRS), even though its presence can only be qualitatively assessed. The NRS consists in a plaque cross-section characterized by a central area of low HU apparently abutting the lumen, surrounded by a ringshaped hyperdense tissue. Because of its qualitative nature, identification of the NRS depends on clinical experience and, therefore, it is affected by a certain degree of interreader variability.

CCTA scans can offer information not only at visual inspection, but also via the use of dedicated coronary segmentation software which can extract volumetric information regarding plaque composition. Quantitative plaque assessment can provide additional information which can help stratifying risk and predicting later outcome $(22,23)$. Hell et al. conducted retrospective casecontrol study to investigate whether quantitative plaque characterization could predict cardiac death in long-term follow-up. The study included a population of 64 patients (32 cardiac death; 32 controls) who underwent CCTA for the inspection of the entire coronary tree in consideration of the diffuse nature of CAD; data sets were then analysed through a semi-automated software which quantified volumetric plaque characteristics. Quantitative measure 
included: total plaque volume, volumes of non-calcified plaque (NCP), low-density non-calcified plaque (LD-NCP; attenuation $<30 \mathrm{HU})$, calcified plaque $(\mathrm{CP})$, plaque burden (percentage of plaque volume over total vessel volume), luminal stenosis and contrast density difference (CDD, a recently introduced normalized measure of luminal contrast kinetics). Results showed that a number of quantitative features could predict cardiac death in long-term followup, including total, non-calcified, and low-density plaque volumes as well as CDD. In particular, the Authors stress the fact that overall plaque burden, rather than individual lesions, holds important prognostic information. In this respect, they argue that software-based quantitation offers an objective assessment of the total plaque burden that is not possible to achieve through visual analysis alone (24).

Another feature detectable in CCTA which appears to correlate with an increased risk of MACE is the alteration of pericoronary adipose tissue (PCAT), which has recently been shown to reflect plaque inflammation at histology (25). Goeller et al. conducted a study to investigate whether PCAT attenuation surrounding the proximal right coronary artery correlated to progression of coronary plaque burden, quantitatively analyzed using semi-automated coronary segmentation software in CCTA. Quantitative volumetric analysis included: total plaque (TP), calcified plaque (CP), noncalcified plaque (NCP), low-density non-calcified plaque (LD-NCP) and corresponding burden (plaque volume $100 \% /$ vessel volume). Results showed that an increase in PCAT attenuation correlates with an increase in NCP, LDNCP and TP burden, but not with CP burden; the Authors therefore suggest that PCAT attenuation may represent a biomarker of high-risk plaque progression which may aid in monitoring plaque progression in medical therapy (26).

The clinical utility of CCTA has been tested in a number of randomized trials, such as the PROMISE (Prospective Multicenter Imaging Study for Evaluation of Chest Pain) and SCOT-HEART (Scottish Computed Tomography of the HEART), among others. Overall, there is evidence that the integration of CCTA analysis in the assessment of stable chest pain can improve clinical outcomes in the follow-up of patients affected by CAD $(15,27)$. In particular, it has been demonstrated that CCTA can aid in the identification of candidates for medical therapy (especially based on statins and antiplatelet medication) or revascularization $(28,29)$.

Besides, CCTA represents a viable tool to appraise and monitor the effect of medical therapy on CAD. In this regard, a prospective multinational study (PARADIGM) enrolled 1,255 patients (statin-naive $v s$. statin-taking) who underwent serial CCTA in order assess the impact of statins on individual coronary atherosclerotic lesions. Plaques were quantitatively analyzed taking into account the following parameters: luminal stenosis, percent atheroma volume $(\mathrm{PAV})$, plaque composition and the presence of high-risk plaque (HRP) features (defined as the presence of lowattenuation plaques, positive arterial remodeling, spotty calcifications). Results showed that while statin therapy did not affect the degree of luminal stenosis, it appeared to induce phenotypic plaque transformation; in particular, an association was found with: slower progression of overall plaque volume, increased plaque calcification and reduction of HRP features (30).

Several Authors demonstrated that the number of vessels involved stands in proportional relation to the hazard ratio (2). This evidence opens the scenario to a more holistic approach to CAD risk assessment, which should account not only for stenosis or single plaque features, but also for the general extent and burden of CAD (31). In this respect, a number of scores have been proposed to quantify plaque burden on CCTA, such as: the segment stenosis score (SSS) and the segment involvement score (SIS) developed by Min et al.; the 3-vessel score (left anterior descending, left circumflex, right coronary), the Duke CAD Index, the CONFIRM risk score, the Leaman score and the SYNTAX score (32), among others. Overall, the concept that emerges from recent research is that a reliable appreciation of cardiovascular risk should include as many parameters as possible, which encompass plaque localization, stenosis, morphology, composition and vulnerability features.

\section{Limitations in conventional CT analysis and future directions}

In recent years CCTA has emerged as a key player in primary/secondary prevention of coronary atherosclerosis thanks to its potential to identify and describe plaques with a diagnostic accuracy comparable to that of invasive techniques.

However, the diagnostic potential of CCTA is burdened by a certain degree of subjectivity and inter-reader variability. Moreover, it is unable to detect some fine elements characteristic of high risk plaques, such as macrophage activity, neovascularization, plaque rupture and plaque erosion, which in turn can all be identified by OCT (33).

Even so, recent technological advances have demonstrated how CCTA can be pushed much beyond the boundaries of subjective anatomical interpretation of 
images, thanks to the wealth of information that can be extrapolated from post-processing quantitative analysis. Indeed, radiological images can be viewed as vast $3 \mathrm{D}$ datasets which, via an appropriate coronary segmentation software, can translate volumetric information into minable datasets. The process of extracting quantitative data from radiological scans goes under the name of radiomics, a field which is bound to have tremendous impact on medical imaging in the future. Its relevance also stems from the fact that these systems incorporate multi-dimensional databases which can integrate patients' clinical and genetic information. As a result of such holistic approach, risk assessment can be conducted not only at a population-based level, but it can also be tailored around the single patient. Such comprehensive approach has shown good results in the field of oncology, but to present date its application to cardiovascular imaging is still scarce (32).

\section{Artificial intelligence: a new era?}

Radiomics represents a newly emerging area of research which aims at extracting more information from scans in terms of quantitative data than it may be feasibly possible to the human eye. This huge amount of data can then be processed via analytic systems which seek to artificially reproduce human intelligence, namely machine learning (ML) and deep learning (DL) techniques. The rationale behind these novel technologies is to identify distinctive imaging features that may aid in the diagnosis, predict prognosis and guide personalized therapy for various conditions. The following paragraphs will provide a brief focus into the topics of radiomics, machine learning and deep learning.

\section{Radiomics}

Radiomics is a technique that allows for precise phenotyping of pathological findings identified in radiological imaging. The concept behind this technology is, roughly speaking, to transform images into data. Radiomic parameters can be sub grouped into four categories: first-order, high-order, shape-based and transform-based (21).

First order parameters basically describe the distribution of $\mathrm{HU}$ values within a target area (mean $\mathrm{HU}$, minimum $\mathrm{HU}$ or standard deviation $\mathrm{HU}$ ), deriving statistical inference from these data. Any aspect regarding spatial information is disregarded in first order parameters, with explains why very diverse plaques may display similar values.
In order to overcome the limitations of first order parameters, high-order features were introduced, which enable the characterization of the texture and heterogeneity of a target lesion via the analysis of spatial voxel distribution. Such goal is achieved by quantifying the spatial co-occurrence of given voxel values. There exist several matrices at hand, each one capable of identifying diverse patterns of spatial voxel distribution. For instance, the gray level co-occurrence matrix (GLCM) identifies areas where voxel pairs of a given value co-occur next to each other: areas where dissimilar voxels frequently co-occur are described as heterogeneous. Similarly, the gray level run length matrix (GLRLM) pinpoints voxels of identical value which repeat along a given axis (Figure 2).

Shape-based parameters focus on aspects of geometrical complexity. For example they describe the surface, volume, compactness (a measure of the surface to volume relation) or fractal dimension (self-repeating patterns), offering somehow a quantitative appraisal of spatial complexity.

Transform-based radiomic features translate information from the spatial domain into the frequency domain. The resulting data can then be further analyzed or used to filter out specific information from the images (34).

\section{$M L$}

The amount of medical data has increased exponentially in recent years and the common goal for researchers has become to find ways to analyze these data in order to extrapolate information of clinical significance. Conventional statistics, based on probability models and designed for population-based analysis, are substantially unsuitable to the task of providing case-specific information and guide clinical decisions. Human intelligence surpasses conventional statistical formulas in identifying unique patterns or inferring complex relationships between data through observation and experience. The aim of artificial intelligence (AI) models is that of recreating these learning patterns and, ideally, think and act humanly. Machine learning (ML), a subcategory of AI, is an analytic method based on computer algorithms that has the ability to learn from previously fed data autonomously. The greater the amount of input from the environment, the better ML algorithms performs: predictions are based on comparisons between a new instance and a previous similar occurrence. Let us briefly describe a few ML algorithms by way of example. Supervised learning algorithms are used to assign a certain classification to a newly encountered instance, either 
Pipeline for the calculation of GLRLM

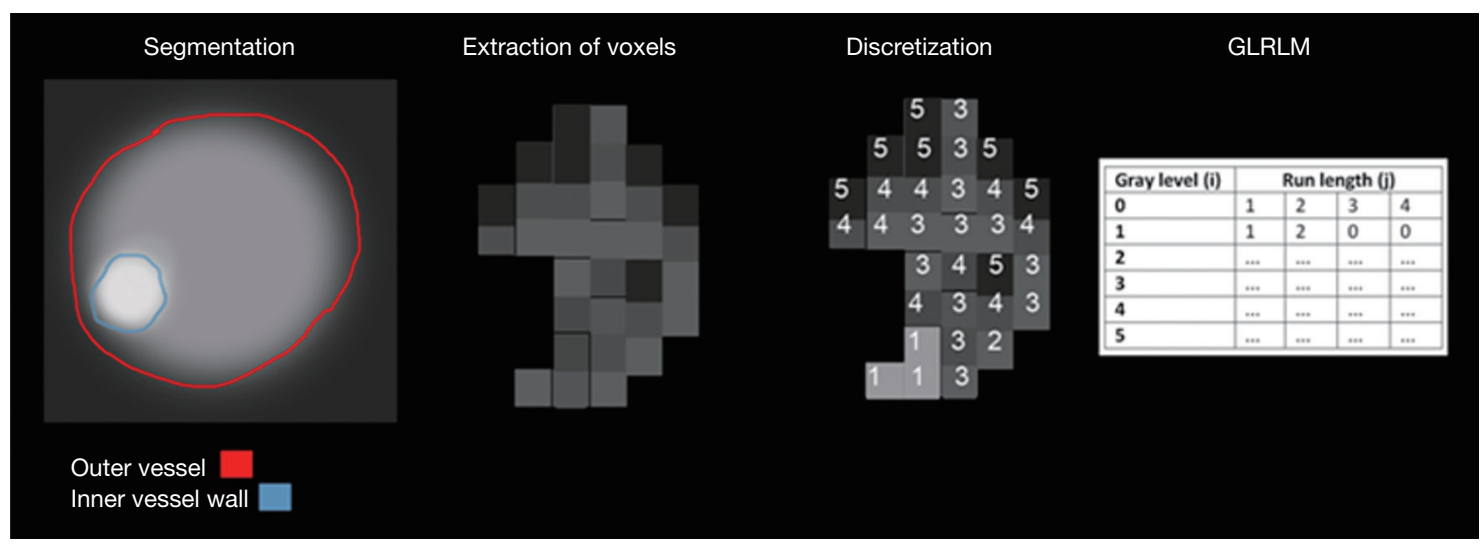

Figure 2 The illustration schematically represents the pipeline for the calculation of GLRLM. First, coronary segmentation takes place, followed by the extraction HU values and discretization into $n$ value groups. Then, for a given direction (i.e., angle 0 degrees), GLRLMs are calculated by reporting the number of times $i$ values appears next to each other in the direction examined. Rows contain the value $i$ (reflecting voxel value) and columns contain the value $j$ (reflecting the number of repetitions in the given direction). The statics are then calculated in various directions and averaged. Readapted from Kolossvary et al. (21).

using bi-modal (i.e., healthy/unhealthy) or continuous (i.e., Agatston score) values. A commonly used algorithm of this type is the so-called k-nearest neighbor, which relies on the basic idea that similar things exist in close proximity. The $\mathrm{k}$ values, which the new occurrences are compared against, are referred to as hyperparameters and are predetermined; optimal values must be balanced in order not to create overfitted or under-fitted ML models.

Another frequently used algorithm is the decisiontree model, often used in the creation of guidelines or risk calculation flow charts. In this case, risk factors are first gathered and a subject is assigned to one of two subgroups based on one parameter (for example gender: Male or Female); subsequently, another parameter is used (i.e., age range) and the patient is assigned to a further subgroup; the process is repeated until more homogenous groups are obtained. The so-called information gain (IG) is a uni-variate analytic method which assesses the relative importance of a certain parameter with regards to a given outcome, in disregard of other possible collinearities

Finally, neural networks are mathematical models which aim to conceptually replicate the organization of the biological brain. Neural networks are organized in single perceptrons, algorithms capable of solving complex problems, which can be aligned to other perceptrons to form multiple layers. A number of so-called hidden layers, which receive no input nor give final outputs, can be stacked in the system. When neural networks contain two or more hidden layers, they are defined deep neural networks (DNN), which substantially represent networks of interconnected regression equations: the output of one equation represent the input to another.

Unsupervised learning algorithms (such as the k-means clustering) differ from the supervised methods described above in that they operate with unlabeled data. The goal here is not to assign a value to a new instance but rather group the data into k clusters, which can then be compared and further analyzed to extrapolate new relationships from the data. Semi-supervised learning algorithms, instead, operate with data that is both labeled and unlabeled. They are useful in those cases in which there is too large an amount of data to be labeled. Thanks to the addition of some labeled cases to the model, semi-supervised models usually perform better than fully unsupervised learning, since some preliminary information is provided regarding how many clusters may be identified or what distribution the parameters have in each group.

\section{$D L$}

DL, an analytic method which is derived from ML, expands on the concept of deep neural networks (DNN) and finds application in complex tasks of AI, such as analyzing visual imagery. In DL, the perceptrons of a neural network are 
not fully connected, but some hidden layers are skipped in order to activate a deeper perceptron, in a fashion similar to pyramidal cells in the brain cortex. In other words, they use a hierarchic approach in the analysis of data, recreating complex patterns from the assembly of simple ones.

\section{Radiomics applied to coronary atherosclerosis}

The first area of research to focus on the potentials of radiomics was oncology, and still nowadays the most advanced applications are seen within this medical area (35). While the scientific community has shown growing interest in the fascinating potentialities and novel applications of radiomics, standardized protocols and techniques must be defined in order to provide research groups and clinicians with a consistent and reliable framework for radiomic analysis. In an attempt to reduce the degree of variability among quantitative imaging biomarkers from different centers worldwide, the Radiological Society of North America (RSNA) initiated the Quantitative Imaging Biomarkers Alliance (QIBA), followed shortly after by the European Imaging Biomarkers Alliance (EIBALL) created by the European Society of Radiology (ESR) (27). Within this scenario, chest CT and coronary artery disease represent a strategic target which is currently attracting ever growing attention. The following section will provide an overview on the current applications of radiomics, ML and DL techniques to the study of atherosclerotic coronary disease.

\section{Present and future applications}

One of the main advantages in employing radiomic techniques in the study of coronary atherosclerotic lesions is to overcome the limitations of qualitatively assessed lesions, which result in inter-reader variability. One typical example that depicts this scenario is the identification of the napkin ring sign, an independent prognostic marker for MACE. Kolossváry et al. conducted a study with the specific intent to determine whether radiomic analysis could improve the identification of NRS plaques (36).

They identified 30 NRS plaques on CCTA out of 2,674 patients studied for stable chest pain and matched them with 30 non-NRS controls (comparable for degree of calcification, degree of stenosis, localization and imaging parameters). Case to control comparison was conducted using 8 conventional and 4,440 radiomic parameters. They were able to demonstrate that there were no significant differences among NRS and non-NRS patients with regards to conventional parameters (namely: lesion length, area of stenosis, mean plaque burden, lesion volume, remodeling index, mean plaque attenuation, and minimal and maximal plaque attenuation). Conversely, radiomic parameters displayed a significant difference between the groups; in particular, features incorporating the spatial distribution of voxels (GLCM, GLRLM, and geometry-based parameters) retained a higher predictive value than first-order statistics (which reflect the mere distribution of the intensity values) (Figure 3). It must be highlighted that the study was limited by the small number of patients enrolled and that the true number of NRS is considerably smaller in a real population. However, the Authors showed that radiomic analysis may indeed compete with the diagnostic accuracy of clinical experts. In addition, from the analysis of datasets emerged a number of clusters of information, which suggests that further experimentation in this direction may allow for the identification of new image markers that are currently unknown. The Authors discuss that in the future radiomics could be integrated into currently used workstations to function as a computer-aided diagnostic tool.

As it was previously discussed, a number of invasive imaging modalities, such as IVUS and OCT, have demonstrated distinctive capacity to identify vulnerable plaques in tests validated by histology and clinical assessment. Moreover, NaF18-PET has been shown to effectively identify inflammation and microcalcifications in coronary atherosclerotic plaques. In a future ideal scenario, these methodologies will be substituted by noninvasive imaging modalities with no loss in diagnostic accuracy. In recent times, CCTA has been proposed as a potential candidate in this role, but it has only shown modest correlation with findings of vulnerable plaque identified by IVUS, OCT, or NaF18-PET. However, the extra information that can be extracted from CT scans using radiomic techniques may provide new answers in the near future. Kolossváry et al. conducted a study to address the question whether coronary CTA radiomics could correlate better than conventional CCTA with invasive and radionuclide imaging markers of high-risk plaques (37). In this retrospective analysis, the Authors analyzed 44 plaques from 25 patients using four imaging methodologies: CCTA, NaF18-PET, IVUS and OCT (in the context of invasive coronary angiography) between March and October 2015. For what concerns conventional morphologic features, the study considered: low attenuation plaque, positive remodeling, spotty calcification and napkin-ring sign. With regards to quantitative analysis (based on HU), it 
Identification of Napkin-Ring Sign plaques by Conventional Quantitative Parameters vs. Radiomic Parameters

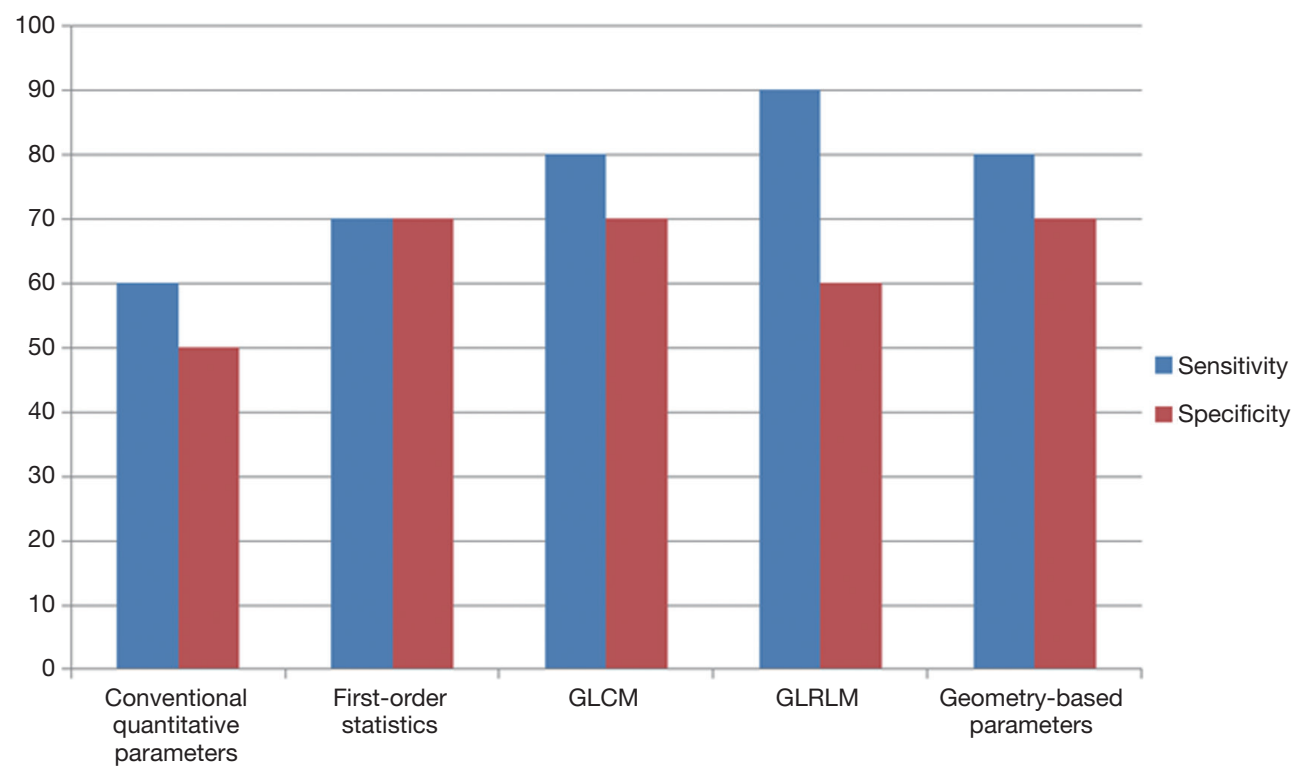

Figure 3 Diagnostic sensitivity and specificity in the identification of plaques with Napkin-Ring Sign by Conventional Quantitative Parameters vs. Radiomic Parameters. The chart graphically represents the results obtained in the study by Kolossváry et al. (the bars represent a rounded off value of the AUC) and it shows that radiomic features were superior in the identification of plaques with napkin ring sign. Conventional quantitative metrics included: mean plaque attenuation; mean plaque burden; lesion volume; minimal plaque attenuation; maximal plaque attenuation; remodeling index; lumen area stenosis; lesion length. GLCM, gray-level co-occurrence matrix; GLRLM, graylevel run-length matrix. Readapted from Kolossváry et al. (32).

considered the volume of: low attenuation non-calcified plaque (<30 HU), non-calcified plaque $(30-130 \mathrm{HU})$, calcified plaque $(>130 \mathrm{HU})$. Finally, the radiomic features accounted for included 935 parameters: 44 first-order statistics; 342 statistics calculated from GLCM; 33 statistics extracted from GLRLM, 516 geometry-based statistical parameters. Overall, the study demonstrated that radiomic analysis performed better than conventional CCTA in identifying specific invasive and radionuclide findings of plaque vulnerability, particularly with regards to features determined via OCT and NaF18-PET.

In another prospective study, Kolossváry et al. analyzed 21 coronary arteries ex vivo obtained from seven male donors with a mean age of 52 . The specimens were studied with CCTA and histologic cross sections. Subsequently, radiomic parameters were extracted to conduct a histogrambased assessment and serve as an input for a machine learning evaluation. At histology, lesions were considered advanced if early fibroatheroma, late fibroatheroma or thin-cap atheroma was found. CT cross sections were classified as homogeneous, heterogeneous or napkin-ring sign positive on the basis of visual assessment. Radiomic parameters included first-order statistics and geometrybased features. With regards to ML, eight models were trained on cross sections selected on a random basis. Visual assessment, histogram-based assessment and the best ML model were then put to the test and compared against the histological findings. In their study, the Authors demonstrate that radiomics-based ML could outperform not only CT angiography, but also the histogram-based measurements in differentiating between early and advanced atherosclerotic lesions (38).

A comparison between machine learning and conventional CCTA was the subject matter of another retrospective study conducted by Masuda et al. in 2018, conducted on 115 patients who underwent both CCTA and IVUS. In this study, the Authors start from the assumption that mean CT number is generally recognized as a useful feature in the characterization of coronary plaques, indicating an optimal threshold of $36 \pm 3 \mathrm{HU}$ to differentiate between fatty- and fibro-fatty plaques. However, the Authors argue that a number of factors limit the reliability of such parameter, 
such as differences in the examination type, in the vessels examined and the CT scanning settings. Therefore, in their study they attempt to verify whether histogram analysis combined with ML can serve as a tool for characterization of coronary plaques with a diagnostic potential superior to median CT number. Despite a number of intrinsic limitations to the study (small sample size, CCTA images obtained with a single protocol and others), the results again lean in favor of the ML performance (39).

\section{Limitations}

While several research groups have demonstrated the potentialities of radiomic analysis, a number of aspects must be taken into consideration to identify its present limitations.

Firstly, radiomic parameters are derived from HU voxel values and, as such, they are limited by all possible factors that may somehow influence their acquisition. These, among others, include: CT hardware, kernel, tube voltage, reconstruction and segmentation algorithms, single patient characteristics. Further data are needed in order to quantify to what extent these variables affect radiomic statistics and, consequently, the generalizability of the radiomic signature of a certain lesion $(21,36)$.

Furthermore, since the number and type of radiomic and machine learning (ML) parameters are arbitrarily decided by the operator, a question arises regarding what the optimal standard should be. An excessively high number of parameters may, for example, result in overfitting, which in turn requires a large number of patients to ensure results of statistical significance (40).

With regards to $\mathrm{ML}$, it must be observed that the diagnostic accuracy of a model should ideally be evaluated by applying it to an external validation cohort. However, since such protocol is technically difficult to implement, in many published studies ML models are run on a whole population and then evaluated on the same population itself by using test-sets and $\mathrm{n}$-fold cross-validation techniques. This approach may eventually lead to biased estimates.

Besides, ML models are designed to makes predictions based on the data on which they were trained. Thus, whenever an ML model happens to be unrepresentative of a given dataset of application, inaccurate predictions will result. This means that ML models are, by their own nature, subject to training biases of some degree.

Another aspect which must be accounted for is that ML models are affected by low diagnostic accuracy in identifying rare cases, since their predictive ability is proportional to the number of similar cases previously encountered. This is in contrast with the human ability to memorize and then recognize the random case even after the experience of few isolated instances (21).

Finally, while radiomic analysis holds a potential as a powerful diagnostic aid in the future, it also threatens to substantially increase the burden of work for the radiologist, especially in consideration of its significant technical complexity. In order for this technology to integrate into the daily clinical routine, further advances are needed in the development of automated, user-friendly and time-saving software platforms which will allow the radiologists to deal with such huge datasets (41).

\section{Conclusions}

Radiomics, machine learning and deep learning are newly emerging imaging techniques which, via the extrapolation of quantitative information from conventional radiologic images, can identify imaging biomarkers that can contribute significantly to the characterization of coronary plaques. Such approach has the potential to overcome the limitations represented by the qualitative interpretation of images and identify unknown disease biomarkers, aiding in diagnostic, prognostic and therapeutical decisions. Several Authors have demonstrated that radiomic analysis and machine learning models can typify coronary atherosclerotic plaques with a level of accuracy comparable, if not superior, to other conventional imaging techniques. However, the amount of clinical data to present date is still scarce and further research is needed in order to standardize radiomic analysis protocols among different centers. Moreover, a number of technical aspects need to be further investigated to ensure the reliability and generalizability of the radiomic features of a given lesion. Similarly, in order to make radiomics a reality in the clinical daily routine, further advances are needed to render such technology sufficiently userfriendly and time effective. Even in consideration of its present limitations, there are great expectations for the future development of radiomics, as this technology has the potential to significantly change the field of medical imaging in the near future.

\section{Acknowledgments}

Funding: None. 


\section{Footnote}

Provenance and Peer Review: This article was commissioned by the Guest Editor (Filippo Cademartiri) for the series "Clinical Impact of Cardiac CT in Clinical Practice" published in Cardiovascular Diagnosis and Therapy. The article was sent for external peer review organized by the Guest Editor and the editorial office.

Conflicts of Interest: All authors have completed the ICMJE uniform disclosure form (available at http://dx.doi. org/10.21037/cdt-20-156). The series "Clinical Impact of Cardiac CT in Clinical Practice" was commissioned by the editorial office without any funding or sponsorship. FC served as the unpaid Guest Editor of the series and serves as an unpaid editorial board member of Cardiovascular Diagnosis and Therapy from Jul 2019 to Jun 2021. LS serves as an unpaid editorial board member of Cardiovascular Diagnosis and Therapy from Jul 2019 to Jun 2021. The authors have no other conflicts of interest to declare.

Ethical Statement: The authors are accountable for all aspects of the work in ensuring that questions related to the accuracy or integrity of any part of the work are appropriately investigated and resolved.

Open Access Statement: This is an Open Access article distributed in accordance with the Creative Commons Attribution-NonCommercial-NoDerivs 4.0 International License (CC BY-NC-ND 4.0), which permits the noncommercial replication and distribution of the article with the strict proviso that no changes or edits are made and the original work is properly cited (including links to both the formal publication through the relevant DOI and the license). See: https://creativecommons.org/licenses/by-nc-nd/4.0/.

\section{References}

1. Barquera S, Pedroza-Tobías A, Medina C, et al. Global Overview of the Epidemiology of Atherosclerotic Cardiovascular Disease. Arch Med Res 2015;46:328-38.

2. Naghshtabrizi B, Moradi A, Amiri J, et al. An Evaluation of the Numbers and Locations of Coronary Artery Disease with Some of the Major Atherosclerotic Risk Factors in Patients with Coronary Artery Disease. J Clin Diagn Res 2017;11:OC21-OC24.

3. Dweck MR, Doris MK, Motwani M, et al. Imaging of coronary atherosclerosis - evolution towards new treatment strategies. Nat Rev Cardiol 2016;13:533-48.

4. Tarkin JM, Dweck MR, Evans NR, et al. Imaging Atherosclerosis. Circ Res 2016;118:750-69.

5. Tonino PA, Fearon WF, De Bruyne B, et al. Angiographic versus functional severity of coronary artery stenoses in the FAME study fractional flow reserve versus angiography in multivessel evaluation. J Am Coll Cardiol 2010;55:2816-21.

6. Owen DRJ, Lindsay AC, Choudhury RP, et al. Imaging of Atherosclerosis. Annu Rev Med 2011;62:25-40.

7. Mangiacapra F, Bressi E, Sticchi A, et al. Fractional flow reserve (FFR) as a guide to treat coronary artery disease. Expert Rev Cardiovasc Ther 2018;16:465-77.

8. Donnino R, Jacobs JE, Doshi JV, et al. Dual-source versus single-source cardiac CT angiography: comparison of diagnostic image quality. AJR Am J Roentgenol 2009;192:1051-6.

9. Carità P, Guaricci AI, Muscogiuri G, et al. Prognostic Value and Therapeutic Perspectives of Coronary CT Angiography: A Literature Review. Biomed Res Int 2018;2018:6528238.

10. Gepner AD, Young R, Delaney JA, et al. Comparison of Carotid Plaque Score and Coronary Artery Calcium Score for Predicting Cardiovascular Disease Events: The Multi-Ethnic Study of Atherosclerosis. J Am Heart Assoc 2017;6:e005179.

11. Thomas IC, Forbang NI, Criqui MH. The evolving view of coronary artery calcium and cardiovascular disease risk. Clin Cardiol 2018;41:144-50.

12. Carità P, Igoren Guaricci A, Muscogiuri G, et al. Prognostic Value and Therapeutic Perspectives of Coronary CT Angiography: A Literature Review. Biomed Res Int. 2018;2018:6528238.

13. Saba L, Saam T, Jäger HR, et al. Imaging biomarkers of vulnerable carotid plaques for stroke risk prediction and their potential clinical implications. Lancet Neurol 2019;18:559-72.

14. Saba L, Yuan C, Hatsukami TS, et al; Vessel Wall Imaging Study Group of the American Society of Neuroradiology. Carotid Artery Wall Imaging: Perspective and Guidelines from the ASNR Vessel Wall Imaging Study Group and Expert Consensus Recommendations of the American Society of Neuroradiology. AJNR Am J Neuroradiol 2018;39:E9-E31.

15. Adamson PD, Newby DE. Non-invasive imaging of the coronary arteries. Eur Heart J 2019;40:2444-54.

16. Tahara N, Kai H, Ishibashi M, et al. Simvastatin attenuates plaque inflammation: evaluation by fluorodeoxyglucose positron emission tomography. J Am Coll Cardiol 
2006;48:1825-31.

17. Tarkin JM, Joshi FR, Evans NR, et al. Detection of atherosclerotic inflammation by 68Ga-DOTATATE PET compared to [18F]FDG PET imaging. J Am Coll Cardiol 2017;69:1774-91.

18. Nørgaard BL, Leipsic J, Gaur S, et al. NXT Trial Study Group. Diagnostic performance of noninvasive fractional flow reserve derived from coronary computed tomography angiography in suspected coronary artery disease: the NXT trial (Analysis of Coronary Blood Flow Using CT Angiography: Next Steps). J Am Coll Cardiol 2014;63:1145-55.

19. Diaz-Zamudio M, Dey D, Schuhbaeck A, et al. Automated Quantitative Plaque Burden from Coronary CT Angiography Noninvasively Predicts Hemodynamic Significance by using Fractional Flow Reserve in Intermediate Coronary Lesions. Radiology 2015;276:408-15.

20. Min JK, Shaw LJ, Devereux RB, et al. Prognostic value of multidetector coronary computed tomographic angiography for prediction of all-cause mortality. J Am Coll Cardiol 2007;50:1161-70.

21. Kolossváry M, De Cecco CN, Feuchtner G, et al. Advanced atherosclerosis imaging by CT: Radiomics, machine learning and deep learning. J Cardiovasc Comput Tomogr 2019;13:274-80.

22. Nadjiri J, Hausleiter J, Jahnichen C, et al. Incremental prognostic value of quantitative plaque assessment in coronary CT angiography during 5 years of follow up. J Cardiovasc Comput Tomogr 2016;10:97-104.

23. Tesche C, Plank F, De Cecco CN, et al. Prognostic implications of coronary CT angiography-derived quantitative markers for the prediction of major adverse cardiac events. J Cardiovasc Comput Tomogr 2016;10:458-65.

24. Hell MM, Motwani M, Otaki Y, et al. Quantitative global plaque characteristics from coronary computed tomography angiography for the prediction of future cardiac mortality during long-term follow-up. Eur Heart J Cardiovasc Imaging 2017;18:1331-9.

25. Antonopoulos AS, Sanna F, Sabharwal N, et al. Detecting human coronary inflammation by imaging perivascular fat. Sci Transl Med 2017;9:eaal2658.

26. Goeller M, Tamarappoo BK, Kwan AC, et al. Relationship between changes in pericoronary adipose tissue attenuation and coronary plaque burden quantified from coronary computed tomography angiography. Eur Heart J Cardiovasc Imaging 2019;20:636-43.
27. Kay FU, Oz OK, Abbara S, et al. Translation of Quantitative Imaging Biomarkers into Clinical Chest CT. Radiographics 2019;39:957-76.

28. Williams MC, Newby DE, Nicol ED. Coronary atherosclerosis imaging by CT to improve clinical outcomes. J Cardiovasc Comput Tomogr 2019;13:281-7.

29. Muniyappa R, Noureldin RA, Abd-Elmoniem KZ, et al. Personalized Statin Therapy and Coronary Atherosclerotic Plaque Burden in Asymptomatic Low/Intermediate-Risk Individuals. Cardiorenal Med 2018;8:140-50.

30. Lee SE, Chang HJ, Sung JM, et al. Effects of Statins on Coronary Atherosclerotic Plaques: The PARADIGM Study. JACC Cardiovasc Imaging 2018;11:1475-84.

31. Bittencourt M S, Hulten E, Ghoshhajra B, et al. Prognostic value of nonobstructive and obstructive coronary artery disease detected by coronary computed tomography angiography to identify cardiovascular events. Circ Cardiovasc Imaging 2014;7:282-91.

32. Kolossváry M, Szilveszter B, Merkely B, et al. Plaque imaging with $\mathrm{CT}$ - a comprehensive review on coronary CT angiography based risk assessment. Cardiovasc Diagn Ther 2017;7:489-506.

33. Nakanishi R, Motoyama S, Leipsic J, et al. How accurate is atherosclerosis imaging by coronary computed tomography angiography? J Cardiovasc Comput Tomogr 2019; 13:254-60.

34. Than JCM, Saba L, Noor NM, et al. Lung disease stratification using amalgamation of Riesz and Gabor transforms in machine learning framework. Comput Biol Med 2017;89:197-211.

35. Gillies RJ, Kinahan PE, Hricak H. Radiomics: Images Are More than Pictures, They Are Data. Radiology 2016;278:563-77.

36. Kolossváry M, Karády J, Szilveszter B, et al. Radiomic Features Are Superior to Conventional Quantitative Computed Tomographic Metrics to Identify Coronary Plaques With Napkin-Ring Sign. Circ Cardiovasc Imaging 2017;10:e06843.

37. Kolossvary M, Park J, Bang J, et al. Identification of invasive and radionuclide imaging markers of coronary plaque vulnerability using radiomic analysis of coronary computed tomography angiography. Eur Heart J Cardiovasc Imaging 2019;20:1250-8.

38. Kolossváry M, Karády J, Kikuchi Y, et al. Radiomics versus Visual and Histogram-based Assessment to Identify Atheromatous Lesions at Coronary CT Angiography: An Ex Vivo Study. Radiology 2019;293:89-96.

39. Masuda T, Nakaura T, Funama Y, Okimotoet al. Machine- 
learning integration of CT histogram analysis to evaluate the composition of atherosclerotic plaques: Validation with IB-IVUS. J Cardiovasc Comput Tomogr 2019;13:163-9.

40. Park JE, Park SY, Kim HJ, et al. Reproducibility and Generalizability in Radiomics Modeling: Possible Strategies in Radiologic and Statistical Perspectives.

Cite this article as: Murgia A, Balestrieri A, Crivelli P, Suri JS, Conti M, Cademartiri F, Saba L. Cardiac computed tomography radiomics: an emerging tool for the non-invasive assessment of coronary atherosclerosis. Cardiovasc Diagn Ther 2020;10(6):2005-2017. doi: 10.21037/cdt-20-156
Korean J Radiol 2019;20:1124-37.

41. Kolossváry M, Kellermayer M, Merkely B, et al. Cardiac Computed Tomography Radiomics: A Comprehensive Review on Radiomic Techniques. J Thorac Imaging 2018;33:26-34. 